\title{
A BEAMFORMING PARTICLE FILTER FOR EEG DIPOLE SOURCE LOCALIZATION
}

\author{
Hamid R. Mohseni, Foad Ghaderi, Edward E. Wilding and Saeid Sanei \\ Schools of Engineering and Psychology \\ Cardiff University, Cardiff, Wales, UK
}

\begin{abstract}
Recently we have developed a method for electroencephalogram (EEG) dipole source localization based on particle filtering (PF). In this study the method is combined with beamforming to eliminate the noise which is spatially uncorrelated with the desired signal and accordingly to improve its performance. The proposed beamforming is an optimum, linear and data independent filter which can be applied to stationary as well as non-stationary data. Simulation and real data results have been provided to show its better performance over PF and beamforming approaches for dipole source localization.
\end{abstract}

Index Terms - Beamforming, dipole source localization, EEG, particle filter.

\section{INTRODUCTION}

EEG is a technique to record electrical activity of the brain and has many clinical, psychological and engineering applications. EEG has an excellent temporal resolution in comparison with other neuroimaging techniques such as funtional magnetic resonance imaging (fMRI). However, EEG suffers from poor spatial resolution and therefore mathematical methods are needed to localize the sources. A popular approach in source localization is dipole source localization, which assumes that one or multiple current dipoles represent the electric sources. The current dipoles are specified with their three dimensional coordinates and their three dimensional moments. The relation between the multichannel measured EEG data $\mathbf{y}$ and the dipole location $\rho$ and moments $\mathbf{m}$ can be given by

$$
\mathbf{y}=\mathbf{F}(\rho) \mathbf{m}
$$

where $\mathbf{F}$ is the gain or lead field matrix and is a non-linear function of the dipole location $\rho$. The matrix $\mathbf{F}$ can be calculated in a spherical or in a realistic head model, where the conductivity and shape of different layers of the head are obtained using imaging systems such as MRI.

Linearly constrained minimum variance (LCMV) beamforming as a spatial filter is a well established method in EEG dipole source localization [1]. LCMV localizes a source based on the output power of a spatial filter constrained to

This work is supported by the Schools of Engineering and Psychology, Cardiff University. minimize the variance at the filter output while passing activity from a location of interest. The transient and possible correlation between the nature of neural activations in different parts of the brain often limits the performance of the LCMV. Since EEG has variable sensitivity to the source location, the noise gain of the filter varies as a function of location. A strategy to account for this effect is to normalize the output variance of the beamformer with respect to the variance estimated at the presence of noise only [2]. Therefore, in beamforming approach, localization of the source is performed by finding a $\rho$ that maximizes

$$
P(\boldsymbol{\rho})=\frac{\operatorname{tr}\left\{\mathbf{F}^{T}(\boldsymbol{\rho}) \mathbf{C}_{y}^{-1} \mathbf{F}^{T}(\boldsymbol{\rho})\right\}}{\operatorname{tr}\left\{\mathbf{F}^{T}(\boldsymbol{\rho}) \mathbf{C}_{n}^{-1} \mathbf{F}^{T}(\boldsymbol{\rho})\right\}}
$$

where $\mathbf{C}_{y}$ is an estimate of the signal covariance and $\mathbf{C}_{n}$ is an estimate of the noise-only covariance.

Other methods including brain electrical source analysis, multiple signal classification (MUSIC) and its modified version, recursively applied and projected MUSIC (RAPMUSIC), also have been documented in the literature. Readers may refer to a recent review in [3] for details.

Recently we proposed a method based on particle filtering (PF) or sequential Monte Carlo techniques for localizing and tracking of EEG dipoles [4]. PF is an emerging methodology which has attracted much attention in different areas of science and engineering. In this study, we design a linear optimum beamforming to be used in PF framework. The method is called beamforming particle filter (BPF) and improves the performance of $\mathrm{PF}$ by eliminating spatially uncorrelated noise components.

\section{METHODS}

\subsection{State space and particle filter}

Consider the following state space

$$
\begin{gathered}
\mathbf{x}_{k}=f_{k-1}\left(\mathbf{x}_{k-1}\right)+\mathbf{w}_{k-1} \\
\mathbf{y}_{k}=h_{k}\left(\mathbf{x}_{k}\right)+\mathbf{v}_{k}
\end{gathered}
$$

where $\mathbf{x}_{k}$ and $\mathbf{y}_{k}$ are the sates and measurements at time $k, f_{k}$ and $h_{k}$ are generally nonlinear functions, and $\mathbf{w}_{k-1}$ and $\mathbf{v}_{k}$ are assumed to be zero mean additive Gaussian white noise 
$(\mathrm{GWN})$ with known covariance matrices $\mathbf{Q}_{w}$ and $\mathbf{Q}_{v}$, respectively.

We search for the filtered estimates of $\mathbf{x}_{k}$ based on a set of available measurements $\mathbf{y}_{1: k}=\left\{\mathbf{y}_{i}, i=1, \ldots, k\right\}$ up to time $k$. When the functions $f_{k}$ and $h_{k}$ are nonlinear or distribution of the state is non-Gaussian, PF has been widely reported as one of the best choices [5]. In PF, the state distribution is approximated by particles $\left\{\mathbf{x}^{(n)}, n=1, \ldots, N\right\}$ and their associated weights $w^{(n)}$. Different methods have been proposed to update current weights based on previous wights and available measurements. However, the most popular one is sampling-importance-resampling (SIR) method. In SIR the weights are easily updated as [5]

$$
w_{k}^{(n)}=w_{k-1}^{(n)} p\left(\mathbf{y}_{k} \mid \mathbf{x}_{k}^{(n)}\right)
$$

If the added noise $\mathbf{v}_{k}$ be GWN, $p\left(\mathbf{y}_{k} \mid \mathbf{x}_{k}^{(n)}\right)$ is equivalent to a zero mean Gaussian distribution with covariance matrix $\mathbf{Q}_{v}$.

\subsection{Dipole source localization formulation in state space}

Suppose the measured multichannel EEG signals $\mathbf{y}_{k}$ from $M$ sensors at time $k$ are produced by $q$ dipoles, so we can model $\mathbf{y}_{k}$ as

$$
\mathbf{y}_{k}=\sum_{i=1}^{q} \mathbf{F}\left(\boldsymbol{\rho}_{k}(i)\right) \mathbf{m}_{k}(i)+\mathbf{v}_{k}
$$

where $\boldsymbol{\rho}_{k}(i)$ is a three dimensional location vector and $\mathbf{m}_{k}(i)$ is a three dimensional moment vector of the $i$ th dipole. We assume the number of dipoles is known a priori and we are interested in estimation of their locations. Hence, our states are defined as

$$
\mathbf{L}_{k}=\left[\begin{array}{lll}
\boldsymbol{\rho}_{k}(1) & \ldots & \boldsymbol{\rho}_{k}(q)
\end{array}\right]
$$

By introducing an $M \times 3 q$ matrix of location $\mathbf{F}\left(\mathbf{L}_{k}\right)$ and a $3 q \times 1$ vector of moments $\underline{\mathbf{m}}_{k}$ as

$$
\begin{gathered}
\mathbf{F}\left(\mathbf{L}_{k}\right)=\left[\begin{array}{llll}
\mathbf{F}\left(\boldsymbol{\rho}_{\mathbf{1}}\right) & \ldots & \mathbf{F}\left(\boldsymbol{\rho}_{\mathbf{q}}\right)
\end{array}\right] \\
\underline{\mathbf{m}}_{k}=\left[\begin{array}{lll}
\mathbf{m}_{k}^{T}(1) & \ldots & \mathbf{m}_{k}^{T}(q)
\end{array}\right]^{T}
\end{gathered}
$$

where $[.]^{T}$ indicates transpose operation, equation (6) can be reformulated in matrix form as

$$
\mathbf{y}_{k}=\mathbf{F}\left(\mathbf{L}_{k}\right) \underline{\mathbf{m}}_{k}+\mathbf{v}_{k}
$$

Here $\mathbf{F}$ is a nonlinear function of $q$ dipoles. Thus, using equation (1), the state space formulations (3 and 4) have the following forms

$$
\begin{gathered}
\mathbf{L}_{k}=\mathbf{L}_{k-1}+\mathbf{w}_{k-1} \\
\mathbf{y}_{k}=\mathbf{F}\left(\mathbf{L}_{k}\right) \underline{\mathbf{m}}_{k}+\mathbf{v}_{k}
\end{gathered}
$$

In the above equation, we still need to know the moments vector $\underline{\mathbf{m}}_{k}$ to estimate $\mathbf{L}_{k}$ using PF. Fortunately, $\underline{\mathbf{m}}_{k}$ has a linear relation with he measured EEG data, and assuming that the moment vector $\underline{\mathbf{m}}_{k}$ is a noiseless process, it can be optimally estimated in each step as

$$
\underline{\mathbf{m}}_{k}=\mathbf{F}^{\dagger}\left(\mathbf{L}_{k-1}\right) \mathbf{y}_{k}
$$

where $\mathbf{F}^{\dagger}\left(\mathbf{L}_{k-1}\right)$ is the pseudo-inverse of $\mathbf{F}\left(\mathbf{L}_{k-1}\right)$ and is given by

$$
\mathbf{F}^{\dagger}=\left(\mathbf{F}^{T} \mathbf{F}\right)^{-1} \mathbf{F}^{T}
$$

Note that in equation (11), $\underline{\mathbf{m}}_{k}$ is estimated from the location matrix $\mathbf{L}_{k-1}$ at previous step and measurements $\mathbf{y}_{k}$ at the current step. The proposed method is a grid based method meaning that the brain is divided into sufficiently small three dimensional grid cells and each dipole's location is restricted to one of these cells. Therefore, after the prediction stage, the locations indicated by each particle may not be one of the grid cells and they need to be replaced with the nearest cells' locations (see Algorithm 1).

\subsection{Linear optimum beamforming}

Consider all of the signals coming from the grid cells $\left\{\mathbf{g}_{i} \mid i=\right.$ $1, \ldots, G\}$ out of which $q$ of them are the sources of interest $\breve{\rho}=\{\boldsymbol{\rho}(i) \mid i=1, \ldots, q\}$, and suppose the recorded EEG can be decomposed as

$$
\mathbf{y}_{k}=\sum_{i=1}^{G} \mathbf{F}\left(\mathbf{g}_{i}\right) \mathbf{m}_{k}(i)
$$

We are looking for a linear spatial filter $\mathbf{W}_{\breve{\rho}}$ which passes signals coming from $\breve{\rho}$ and suppresses the rest. Therefore, the filter $\mathbf{W}_{\breve{\rho}}$ should have the following ideal response

$$
\mathbf{W}_{\breve{\rho}}^{T} \mathbf{y}=\sum_{i=1}^{G} \mathbf{W}_{\breve{\rho}}^{T} \mathbf{F}\left(\mathbf{g}_{i}\right) \mathbf{m}_{k}(i)=\sum_{i=1}^{q} \mathbf{F}\left(\boldsymbol{\rho}_{k}(i)\right) \mathbf{m}_{k}(i)
$$

Equation (14) results in

$$
\mathbf{W}_{\breve{\rho}}^{T} \mathbf{F}\left(\mathbf{g}_{i}\right)= \begin{cases}\mathbf{F}\left(\mathbf{g}_{i}\right) & \mathbf{g}_{i} \in \breve{\rho} \\ \mathbf{O} & \mathbf{g}_{i} \notin \breve{\rho}\end{cases}
$$

where $\mathbf{O}$ is a $3 \times M$ null matrix. Equation (15) can be formulated in a matrix form as

$$
\mathbf{W}_{\breve{\rho}}^{T} \underline{\mathbf{F}}=\mathbf{F}_{\mathbf{O}}
$$

where $\underline{\mathbf{F}}=\left[\begin{array}{lll}\mathbf{F}\left(\mathbf{g}_{1}\right) & \ldots & \mathbf{F}\left(\mathbf{g}_{G}\right)\end{array}\right]$ is the matrix of all gains and $\mathbf{F}_{\mathbf{O}}=\left[\begin{array}{llllll}\mathbf{O} & \ldots & \mathbf{F}(\boldsymbol{\rho}(1)) & \ldots & \mathbf{F}(\boldsymbol{\rho}(q)) \ldots & \mathbf{O}\end{array}\right]$ has zero entries except in the locations of interest. The optimum solution to the linear equation (16) is

$$
\mathbf{W}_{\breve{\rho}}=\underline{\mathbf{F}}^{T \dagger} \mathbf{F}_{\mathbf{O}}^{T}
$$

In the above equation, the pseudo-inverse of $\underline{\mathbf{F}}^{T}$ is computed instead of pseudo-inverse of $\underline{\mathbf{F}}$. This requires computing the inverse of a lower dimension matrix (see equation (12)). By 
applying this filter to the measured EEG we will have a data which suffers from less spatial noises.

This filter, in contrast to other filters such as LCMV, is a data independent filter and there is no need for the second order statistics of the signals. Therefore, it can be applied to the stationary as well as non-stationary signals.

\subsection{Beamforming particle filter}

The idea of BPF is to use the spatially filtered data instead of the original measurements to compute the moment vector $\underline{\mathbf{m}}_{k}$. Therefore, by applying the obtained filter from equation (17) to the measurements, equation (11) is converted to

$$
\underline{\mathbf{m}}_{k}=\mathbf{F}^{\dagger}\left(\mathbf{L}_{k-1}\right) \mathbf{W}_{\breve{\rho}}^{T} \mathbf{y}_{k}
$$

The matrix $\mathbf{F}_{\mathbf{O}}$ in equation (17) is constructed for each particle using locations indicated by particles from previous step. Moreover, since matrix $\mathbf{F}$ is the matrix of all gains and is independent of the desired locations, for computational efficiency, its pseudo-inverse can be calculated once before the algorithm starts. The pseudo-code of this algorithm is presented in Algorithm 1. In this algorithm, $\mathcal{N}\left(\mathbf{O}, \mathbf{Q}_{v}\right)$ means a zero mean Gaussian distribution with covariance matrix $\mathbf{Q}_{w}$.

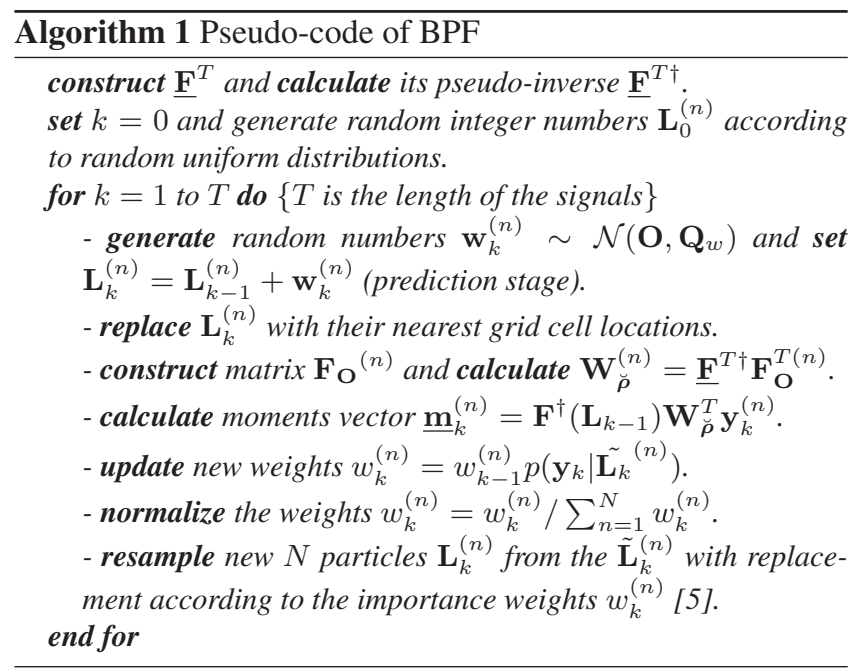

\section{NUMERICAL RESULTS}

In this section, a comparison between the BPF, PF, and beamforming approaches is given. A three shell realistic geometry inhomogeneous head model was used. The methods were applied on a discrete cubic grid with 413 grid cells. A 25electrode configuration was used and the electrodes were assumed to have a homogeneous distribution over the hemispheres according to the standard 10-20 system.

In the first simulation, one stationary dipole located in the superficial region of the brain is considered. The moments in

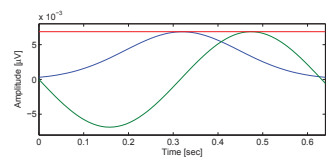

(a)

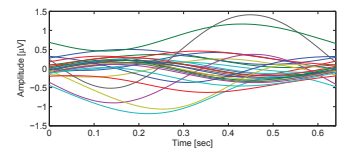

(b)

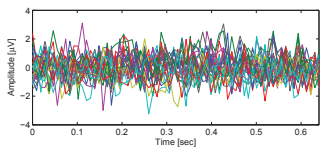

(c)
Fig. 1. An example of simulated data, (a) dipole's moment in $x, y$ and $z$ directions, (b) simulated noiseless EEG, (c) simulated noisy EEG with SNR $=-5 \mathrm{db}$.

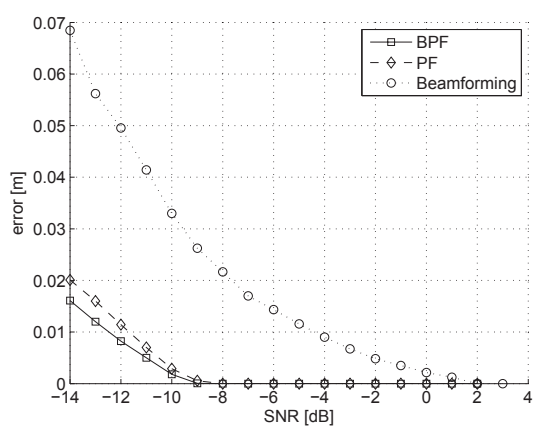

Fig. 2. Output error of three different methods in different values of SNR.

$x, y$ and $z$ directions are assumed to be a sinusoidal, a constant, and a Gaussian function of time, respectively. An example of moments, noiseless and noisy simulated EEG with SNR $-5 \mathrm{~dB}$ are shown in Fig. 1. In both PF and BPF approaches, all the noise covariance matrices $\mathbf{Q}_{w}$ and $\mathbf{Q}_{v}$ are simply assumed to be in the forms of $\sigma_{w} \mathbf{I}$ and $\sigma_{v} \mathbf{I}$ and same number of particles was used for both PF and BPF.

Fig. 2 shows the error of the estimated dipole's location in different signal-to-noise-ratio (SNR) values. The error is defined as the $3 \mathrm{D}$ geometrical distance between the estimated and simulated locations in meter unit. BPF and PF approaches exhibit extremely better performance than the beamforming which is very sensitive to SNR. BPF outperforms PF method and exhibit more robustness to noise. It is noteworthy that the results may change with the number and depth of dipoles as well as the correlation and distance between the dipoles. The results demonstrate the potential use of PF and BPF methods for EEG with very low SNR.

Fig. 3 presents an example for when the assumption on the number of dipoles $q$ is violated. In this example, two dipoles, one in superficial region of right hemisphere and one in the left hemisphere, are used to simulate the data. The first dipole has stronger moment's power than the second one and their locations are shown with blue circles in Fig. 3. We assumed one dipole is responsible for the simulated data and then the above three methods were applied. The result of lo- 


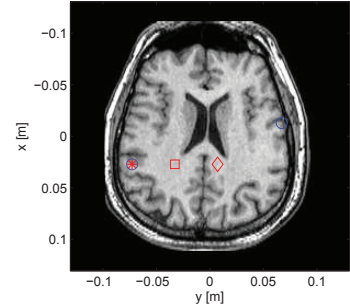

(a)

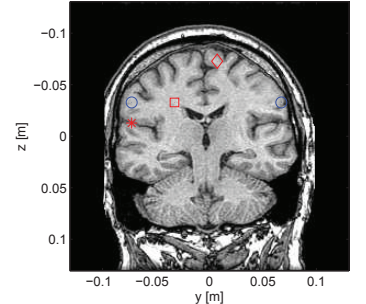

(b)
Fig. 3. Estimating the location of two dipoles with one dipole, the original two locations are shown with circle and estimated locations using BPF, PF and beamforming are dentated by asterisk, square and diamond, respectively, (a) axial view, (b) coronal view.

calization using BPF, PF, and beamforming are presented in Fig. 3 by asterisk, square and diamond, respectively, in axial and coronal views. BPF located the first dipole very close to the strongest dipole and PF located the dipole between the two dipoles but closer to the stronger one. On the other hand, beamforming located the dipole between the two dipoles with no regard to the stronger dipole. This example shows that if the assumed number of dipoles $q$ is less than the number of existing dipoles, then the BPF method reveals the strongest dipoles and the PF finds the locations for those that minimize the error between the constructed data and the original measurements. From physiological point of view, therefore the performance of BPF may be more acceptable.

\section{AN EXPERIMENTAL RESULT}

EEG data were recorded in electrophysiology laboratory of Cardiff university. The sampling frequency was set to $200 \mathrm{~Hz}$ and a linear filter with a bandwidth between 0.03 and $40 \mathrm{~Hz}$ was applied. A $0.15 \mathrm{sec}$ pre-stimulus interval was used for baseline correction. Event related potentials (ERPs) are acquired in the test phase of a memory experiment. In the test phase, the participants were asked to respond on one key to words encountered in a prior study phase (studied words). The number of studied words was set to 70 .

Fig. 4(a) shows the average of ERPs over trials elicited in correct responses to the studied words. The shown ERPs are the superposition of 25 sensors for one subject. The first peak is considered as an exogenous ERP which is related to the visual process of the brain and the second peak is a cognitive related ERP. Fig. 4(b) is the averaged re-referenced and normalized data which is used for processing. Figs. 4(c) and (d) present the results of localization for the first peak in coronal and axial views. The estimated location of the first peak using beamforming, PF and BPF are denoted by square, circle and asterisk, respectively. The location of the first peak which is a visual process is in the primary visual cortex and is located in the back of the brain (parietal site). Both images illustrate that the location obtained by the BPF method is in the parietal site near the primary visual cortex. The location obtained by the
$\mathrm{PF}$ is more accurate than that obtained by the beamforming in coronal view and the location obtained by the beamforming is more accurate than that from the PF in axial view. In this memory experiment, multiple sources are activated and we expect that the BPF to show the location of the strongest sources and the PF method to show the weighted average of all source locations. Thus, in this experiment, BPF approach outperformed PF and beamforming techniques.

\section{CONCLUSION}

In this study, a method for localizing EEG sources based on beamforming and PF was presented. We demonstrated the accuracy of the method for the simulated data along with the real data. The observations proved accurate performance of PF and BPF over beamforming approach. Moreover, BPF not only outperforms PF in different values of SNR, but also if the assumed number of dipoles is violated, BPF has a better performance. The BPF and PF, with the same formulation, can also be applied to magnetoencephalogram (MEG) data.

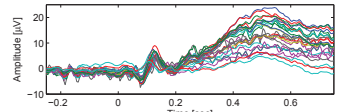

(a)

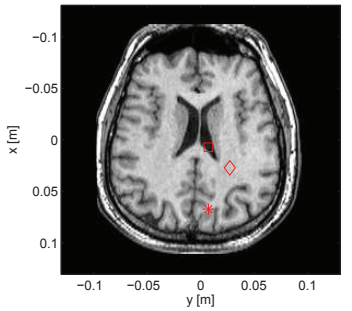

(c)

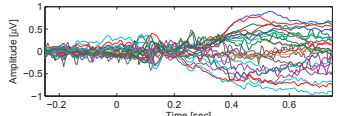

(b)

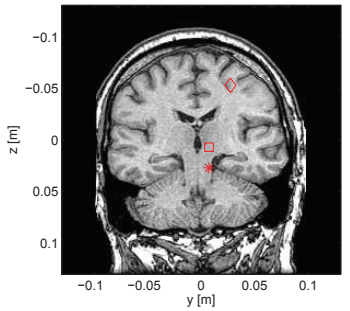

(d)
Fig. 4. An example of applying three methods on real data, (a) superposition of average of ERPs in one subject for 25 electrodes, (b) averageed rereferenced normalized ERPs, (c) estimated locations in axial view using BPF, $\mathrm{PF}$ and beamforming denoted by asterisk, square and diamond, respectively, (d) same results in coronal view.

\section{REFERENCES}

[1] B. D. Van Veen, W. Van Dronglen, M. Yuchtman, and A. Suzuki, "Localization of brain electrical activity via linearly constrained minimum variance spatial filtering," IEEE Trans. Biomed. Eng., vol. 44, pp. 867880, 1997.

[2] S. E. Robinson and J. Vrba, "Functional neuroimaging by synthetic aperture magnetometry (sam)," in Recent Advances in Biomagnetism, T. Yoshimoto, M. Kotani, S. Kuriki, H. Karibe, and N. Nakasato, Eds. 1999, pp. 302-305, Japan: Tohoku Univ. Press.

[3] S. Baillet, J. C. Mosher, and R. M. Leahy, "Electromagnetic brain mapping," IEEE Signal Processing Magn., vol. 18, pp. 14-30, 2001.

[4] H. R. Mohseni, E.L. Wilding, and S. Sanei, "Sequential monte carlo techniques for eeg dipole placing and tracking," in Proc. The 5th IEEE Sensor Array and Mutichanel Signal Process. workshop (SAM), July 2008.

[5] A. Doucet, N. Freitas, and N. Gordon, Sequential monte carlo metods in practice, Springer, 2001. 